\title{
VALORACIÓN ECONÓMICA DE LOS BENEFICIOS AMBIENTALES DE LA RECUPERACIÓN DEL RÍO SEGURA (ESPAÑA)*
}

\author{
A. Perni** \\ J. M. Martínez-Paz ${ }^{* *}$ \\ Recibido: mayo 09 de 2012 • Aceptado: noviembre 13 de 2012
}

\section{RESUMEN}

La Directiva Marco del Agua de la Unión Europea establece la necesidad de alcanzar el buen estado ecológico de las masas de agua para el año 2015. Esto requiere la aplicación de diferentes medidas, entre las que destacan los planes de restauración ambiental de ríos. Además, esta Directiva integra el uso de instrumentos económicos para justificar aquellas medidas diseñadas para conseguir el buen estado de una masa de agua. Este trabajo tiene como objetivo valorar en términos monetarios los beneficios ambientales de la recuperación ambiental de un ecosistema natural, el río Segura, mediante el método de valoración contingente. Los resultados muestran que los beneficios generados por el río tras su recuperación ascenderían a los siete millones de euros anuales. Este valor debería ser considerado en los análisis costo-beneficio de las actuaciones emprendidas en el río Segura con el fin de justificar su rentabilidad social desde una óptica económica.

\section{PALABRAS CLAVE}

Bienes y servicios ambientales, restauración de ríos, valoración contingente, economía ambiental

\section{CLASIFICACIÓN JEL}

Q51, 053, Q58

\section{CONTENIDO}

Introducción y objetivos; 1. Marco teórico general; 2. Caso de estudio: el río Segura en su tramo Ojós-Contraparada; 3. Diseño de la Valoración Contingente; 4. Resultados; 5. Conclusiones; Bibliografía.

\footnotetext{
Este artículo de investigación es resultado del proyecto "Aspectos económicos de la planificación hidrológica en cuencas mediterráneas" financiado por la Fundación Instituto Euromediterráneo del Agua (2009-2010), y del proyecto GEAMED sobre "Gestión y eficiencia del Uso Sostenible del agua de Riego en la Cuenca mediterránea. Sureste" (AGL2010-22221-C02-01), cofinanciado por el Ministerio de Ciencia e Innovación español y el Fondo Europeo de Desarrollo Regional (2010-2013). Durante el desarrollo de este trabajo, Ángel Perni disfrutó de una beca predoctoral concedida por la Fundación Séneca de la Región de Murcia (España).

"* Licenciado en Ciencias Ambientales, Universidad de Murcia, Murcia, España. Máster en Gestión y Administración del Agua, Universidad de Murcia, Murcia, España. Becario de Investigación del Departamento de Economía Aplicada, Facultad de Economía y Empresa, Campus de Excelencia Internacional Regional "Campus Mare Nostrum", Universidad de Murcia 30100, Murcia, España. Teléfono: + 3486888 3374. Correo electrónico: angel.perni@um.es.

*.* Ingeniero Agrónomo, Universidad de Córdoba, Córdoba, España. Doctor en Ciencias Económicas, Universidad de Murcia, Murcia, España. Profesor Titular del Departamento de Economía Aplicada, Facultad de Economía y Empresa, Campus de Excelencia Internacional Regional "Campus Mare Nostrum", Universidad de Murcia 30100, Murcia, España. Teléfono: + 3486888 7931. Correo electrónico: jmpaz@um.es.
} 


\section{ECONOMIC VALUATION OF THE ENVIRONMENTAL BENEFITS IN THE RECOVERY OF SEGURA RIVER (SPAIN)}

\section{ABSTRACT}

The European Union Water Framework Directive establishes the need to reach a good ecological status of water bodies by 2015. This requires the application of different measures, among which the plans for environmental restoration of rivers stand out. Furthermore, this Directive integrates the use of economic instruments to justify those measures designed to achieve a proper state of the water body. The objective of this study is to evaluate in monetary terms the environmental benefits that the environmental restoration of a natural resource such as Segura River brings by using the contingent valuation method. The results evidence that the benefits generated by the river after its recovery would exceed 7 million euros per year. This value should be considered in the cost-benefit analysis for the actions taken in Segura River in order to justify its social profitability form an economic standpoint.

\section{KEY WORDS}

Environmental goods and services, river restoration, Contingent valuation, Environmental Economy

\section{JEL CLASSIFICATION}

Q51, Q53, Q58

\section{CONTENT}

Introduction and objectives; 1. General theoretical framework; 2. Case study; Segura River in the Ojos-Contraparada sector; 3. Contingent valuation design design; 4. Results; 5 . Conclusions; Bibliography.

\section{VALORAÇÃO ECONÓMICA DOS BENEFICIOS AMBIENTAIS DA RECUPERAÇÃO DO RÍO SEGURA (ESPAÑHA)}

\section{RESUMO}

A Diretiva Marco de Agua da União Européia estabelece a necessidade de alcançar o bom estado ecológico das massas de agua para o ano 2015. Isto requer a aplicação de diferentes medidas, entre as que se destacam os planos de restauração ambiental de rios. Além disso, esta Diretiva integra o uso de instrumentos económicos para justificar aquelas medidas desenhadas para conseguir o bom estado de uma massa de agua. Este trabalho tem como objetivo valorar em termos monetários os benefícios ambientais da recuperação ambiental de um ecossistema natural, o Rio Segura, por meio do método de valoração contingente. Os resultados mostram que os benefícios gerados pelo Rio após sua recuperação ascenderiam aos sete milhões de euros anuais. Este valor deveria ser considerado nos análises custo-benefício das atuações empreendidas no Rio Segura com o fim de justificar sua rentabilidade social desde uma ótica económica.

\section{PALAVRAS CHAVE}

Bens e serviços ambientais, restauração de rios, Valoração Contingente, Economia Ambiental

\section{CLASIFICAÇÃO JEL}

Q51, Q53, Q58

\section{CONTEUDO}

Introdução e objetivos; 1. Marco teórico geral; 2. Caso de estudo: o Rio Segura em seu tramo Ojos-Contraparada; 3. Desenho da Valoração Contingente; 4.Resultados; 5. Conclusões; Bibliografia. 
Valoración económica de los beneficios ambientales de la recuperación del río Segura (España)

\section{INTRODUCCIÓN Y OBJETIVOS}

La degradación de la estructura y del funcionamiento de los ecosistemas acuáticos es una realidad estudiada y documentada en todo el mundo (Sari y otros, 2003; Fianko, Osea y Anchel, 2008; EEA, 2009). Así, con el objetivo de eliminar o, al menos, mitigar los impactos de la actividad humana sobre estos ecosistemas, muchos países han elaborado normas cuyo objetivo es proteger y conservar sus aguas (Bouleau, 2008). En el caso de la Unión Europea este interés se manifiesta en la Directiva Marco del Agua (DMA), cuyo objetivo es conseguir un buen estado ecológico de las aguas comunitarias para el año 2015 (Comisión Europea, 2000).

El enfoque conservacionista de la DMA aboga por el uso combinado de criterios biológicos, físico-químicos e hidromorfológicos en la definición del buen estado de una masa de agua (Ortiz, 2002). Con el fin de cumplir las obligaciones emanadas de la DMA, se han realizado esfuerzos para mejorar la calidad de las aguas mediante el control y depuración de los vertidos. En la actualidad, dado que el mero cumplimiento de los parámetros físico-químicos preestablecidos no es el único objetivo de la DMA, sino la protección y conservación de los ecosistemas acuáticos, se han desarrollado numerosos proyectos de restauración ambiental y también, aunque menos ambiciosos en cuanto a sus objetivos, de recuperación ambiental de ríos (Skinner y Bruce-Burgess, 2007). Preservar la estructura y dinámica de los ecosistemas fluviales permite mantener en el largo plazo los servicios ambientales prestados por los mismos y, por tanto, contribuye a mejorar los niveles de bienestar social (Klessig, 2001).

La DMA establece también la necesidad de incluir en la gestión de los sistemas hídricos otro tipo de criterios, entre los que se incluyen los instrumentos económicos. De este modo, en el articulado de esta Directiva queda especificado que para la gestión de las cuencas hidrográficas se ha de realizar un análisis económico de los usos del agua (art. 5); conseguir la recuperación de costos de los servicios del agua, entre los que se deben incluir los costos ambientales y del recurso (art. 9), y seleccionar un Programa de Medidas para conseguir el buen estado ecológico de las masas de agua diseñado a partir de análisis costo-efectividad (art. 11) (Comisión Europea, 2003).

Desde la aprobación de la DMA allá por el año 2000, la necesidad de dar respuesta a sus preceptos ha promovido el desarrollo de disciplinas como la ecología, la cual ha experimentado importantes avances en la determinación de indicadores del estado ecológico de las aguas y cálculo de caudales ecológicos (Ivesa, Lyons y Devescovi, 2009), así como en el diseño de proyectos de restauración de ríos (Newson y Large, 2006; England, Skinner y Carter, 2008; Mouton y otros, 2009). No obstante, 
en el campo de la economía los progresos han sido un tanto más desiguales, dada la indeterminación de algunos de los aspectos económicos de la DMA como, por ejemplo, la definición de beneficio y costo ambiental (Bateman y otros, 2006; Schaafsma y Brouwer, 2006; Strosser, 2006; Martín-Ortega, 2013).

El presente trabajo tiene como objetivo valorar en términos monetarios los beneficios ambientales resultantes de la recuperación de un ecosistema fluvial. El caso de estudio se sitúa en el río Segura (España) a su paso por el tramo conocido como Ojós-Contraparada, en el que hasta el día de hoy se han llevado a cabo diversas tareas de recuperación ambiental y revalorización de los elementos culturales asociados al río (BOE, 2001 y 2004). Para ello se ha aplicado el método de la Valoración Contingente (VC), profusamente empleado en la valoración socioeconómica de los elementos que integran el medioambiente.

Trabajos como el aquí presentado tienen una especial utilidad para la gestión sostenible de los recursos hídricos, pues posibilita, de un lado, la inclusión del análisis económico-ambiental en la evaluación de este tipo de proyectos y, de otro, una vía para la participación pública (Ciriacy-Wantrup, 1952; Mitchell y Carson, 1989; Azqueta, 2002; Cardoso y Benhin, 2011). De esta manera, la monetarización de los beneficios ambientales generados por la restauración de las masas de agua permite justificar las inversiones realizadas por las administraciones públicas en un escenario de restricción presupuestaria. Así, en este trabajo se ha obtenido el valor de renta ambiental que el río Segura generaría tras su recuperación, el cual debería ser incorporado a los análisis costo-beneficio de las actuaciones emprendidas para ver si están o no justificadas desde un punto de vista del bienestar social.

Como antecedentes de la aplicación de la VC a ecosistemas acuáticos cabe citar el trabajo de Lant y Mullens (1992), pioneros en aplicar este método a ríos y lagos para estimar la disposición a pagar por una mejora de la calidad de las aguas. En la misma línea, cabe destacar los trabajos de Brox, Kumar y Stoltery (2003) y Cooper, Poe y Bateman (2004). Más reciente es el trabajo de Moreno-Sánchez y otros (2012), que han estimado el valor de un pago por servicios ambientales para la protección de una cuenca andina colombiana a través del método de VC, con la novedad de que dicho pago es variable en función de las características socioeconómicas de los usuarios de la cuenca. En el contexto de la DMA, en el que se desarrolla este trabajo, Brouwer (2004) estima los beneficios públicos del buen estado ecológico de las aguas en el río Scheldt mediante VC. También Birol y otros (2006) y Bateman y otros (2006) centran sus trabajos en las implicaciones de la DMA. En España, Martín-Ortega (2009) lleva a cabo un estudio en el río Guadalquivir para analizar los beneficios ambientales de la mejora de la calidad de sus aguas. Por otra parte, Del 
Valoración económica de los beneficios ambientales de la recuperación del río Segura (España)

Saz-Salazar, Hernández y Sala (2008) estudian la disposición a pagar por la mejora de la calidad del agua del río Serpis en la cuenca del Júcar (España). Finalmente, Perni, Martínez-Carrasco y Martínez-Paz (2011) estiman la disposición a pagar por la mejora del estado ecológico de la masa de agua Mar Menor (España).

Este trabajo está dividido en cinco secciones, sin incluir la presente introducción. La primera sección presenta el marco teórico general de la valoración económica de bienes y servicios ambientales, centrada en los ecosistemas fluviales y el método de valoración contingente. En la segunda, se describen los principales problemas del área de estudio, así como las actuaciones de recuperación ambiental previstas en el mismo. En la tercera, se presenta el diseño de la valoración contingente aplicada para la estimación de los beneficios económicos derivados de la mejora del río Segura. En la cuarta parte se muestran los resultados del estudio y, por último, en la quinta parte, las conclusiones.

\section{MARCO TEÓRICO GENERAL}

Los beneficios que la sociedad recibe de los ecosistemas fluviales dependen del estado de conservación de los mismos y se derivan de las cuatro esenciales funciones que cumplen (Brauman y otros, 2007). Los ecosistemas fluviales tienen una función de abastecimiento de agua y materias primas; una función de regulación de los procesos naturales, como protección frente a avenidas o amortiguación de la contaminación; también cumplen con una función cultural derivada del patrimonio histórico ligado a estos ecosistemas y de sus valores estéticos y paisajísticos; por último, una función de soporte que se corresponde con aquellos procesos propios del ecosistema que sustentan los tres servicios anteriores. Las tres primeras funciones confieren a los ecosistemas un innegable valor, cuya existencia está ligada a la función de soporte. Dicho valor queda fuera de ideas meramente crematísticas y engloba aspectos tan amplios que incluyen tanto el medio natural como el medio socioeconómico.

La figura 1 muestra cómo cada una de las funciones descritas se corresponde con distintos tipos de valor que, de manera conjunta, determinan el Valor Económico Total (VET) de un ecosistema (Pearce y Turner, 1995). Existe dos grandes categorías: valor de uso y valor de no uso, subdividas, a su vez, en otras. Como explica Azqueta (2002), el valor de uso directo hace referencia a aquellos elementos del medio natural que pueden ser utilizados por el hombre (por ejemplo, peces), mientras que el valor de uso indirecto tiene que ver con aquellos elementos de los que depende el anterior (por ejemplo, plancton). El valor de opción es aquel que tiene un elemento natural sin uso actual pero que, quizá, sí lo tenga en un futuro. En ocasiones, este tipo de valor es considerado como un valor independiente del resto o, incluso, como un 
valor de no uso. La última de estas categorías está formada por el valor de existencia y legado que todo ecosistema posee por el mero hecho de existir y por la posibilidad de darlo en herencia a generaciones futuras. En la tabla 1 se clasifican distintos elementos y aspectos de los ecosistemas fluviales en función de la categoría de valor a la que pertenecen.

Figura 1. Funciones básicas de los ecosistemas, y su relación con cada uno de los valores que componen su valor económico total.

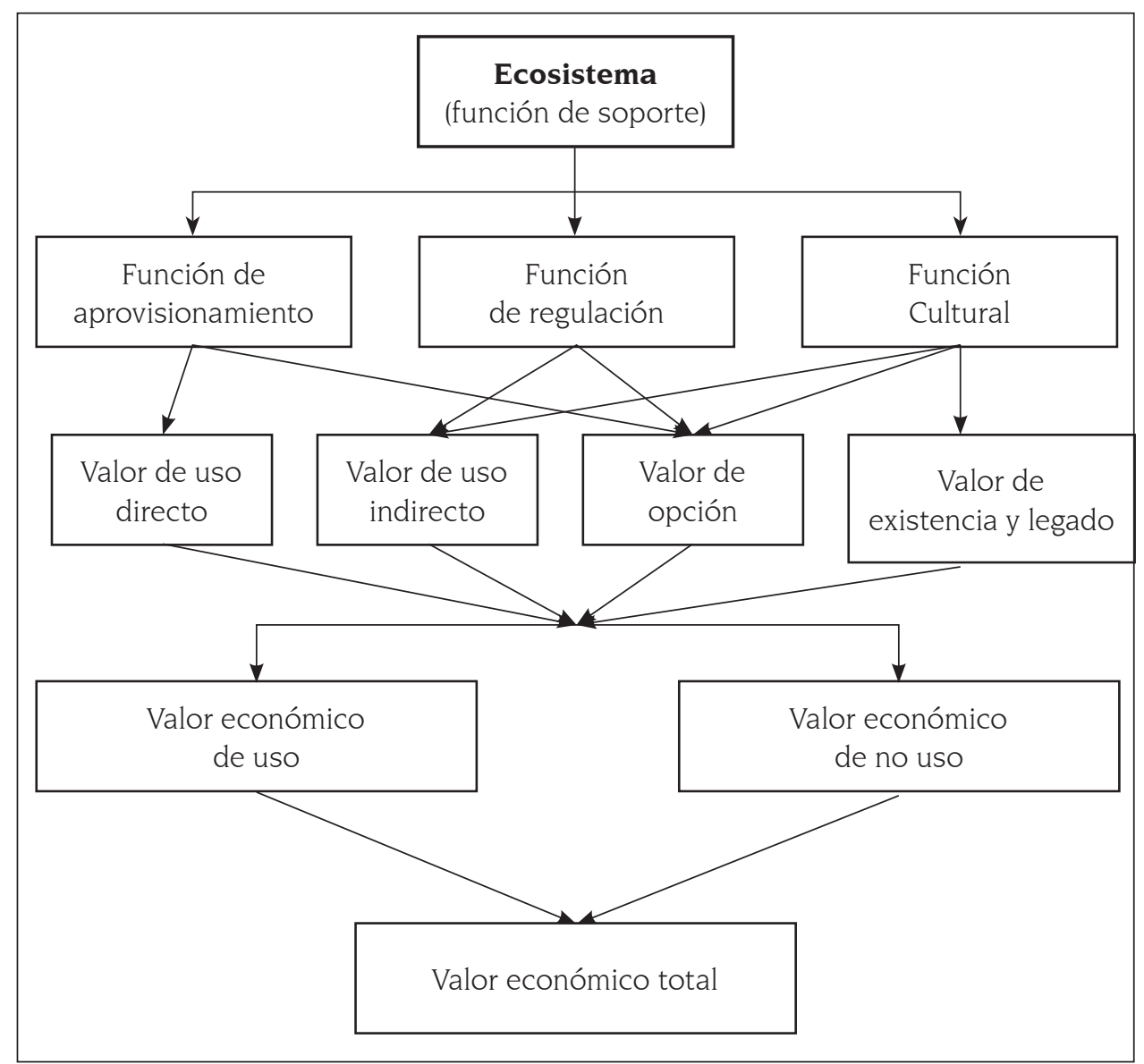

Fuente: Tomada de Hein y otros, 2006, p. 211).

El desconocimiento de este tipo de valores y la falta de una unidad homogénea de valoración hace que el VET sea excluido en la toma de decisiones que afectan de manera directa el estado de conservación de un ecosistema (Azqueta, 1997). Como se verá en la siguiente sección, el caso del río Segura es un fiel ejemplo de esto. 
Valoración económica de los beneficios ambientales de la recuperación del río Segura (España)

Tabla 1. Componentes del valor económico total de un ecosistema fluvial.

\begin{tabular}{|l|l|l|l|}
\hline \multicolumn{2}{|c|}{ Valores de uso } & \multicolumn{2}{c|}{ Valores de no uso } \\
\hline \multicolumn{1}{|c|}{ Directos } & \multicolumn{1}{|c|}{ Indirectos } & \multicolumn{1}{c|}{ Opción } & \multicolumn{1}{c|}{ Existencia } \\
\hline Pesca & Cadena trófica & Posibles usos & Biodiversidad \\
Agricultura & Retención de & $\begin{array}{l}\text { futuros (directos e } \\
\text { indirectos) }\end{array}$ & $\begin{array}{l}\text { Cultura y } \\
\text { patrimonio }\end{array}$ \\
Leña & nutrientes & Información útil & Valor de legado \\
Recreación & Control de & & \\
Transporte & crecidas e & & \\
Energía & inundaciones & & \\
Flora y fauna & Recarga de & & \\
silvestres & acuíferos & & \\
\hline
\end{tabular}

Fuente: tomada de Barbier, Acreman y Knowler (1997, p. 16).

La necesidad de cuantificar el VET de los ecosistemas fluviales viene motivada por el actual estado de degradación que presentan muchos de ellos (EEA, 2010). Para evitar esto, desde la disciplina de la economía ambiental se han diseñado distintos métodos para la valoración de los bienes y servicios provistos por los ecosistemas naturales (Freeman, 2003). Uno de estos métodos es la VC, técnica empleada para estimar el valor de bienes o servicios para los que no existe mercado, como el caso de ciertos elementos integrantes del medioambiente. Esta técnica se basa en la simulación de un mercado hipotético mediante encuestas en las que se pregunta por la disposición máxima a pagar por un bien o servicio ambiental a sus usuarios potenciales (Pearce y Özdemiroglu, 2002). De esta manera es posible estimar el valor de un activo ambiental en términos monetarios, indicador de la contribución de dicho activo a los niveles de bienestar social.

Una forma de medir este bienestar mediante el método de VC es a partir de la variación equivalente en la calidad del activo ambiental, enfoque empleado en este trabajo. Se trata de un escenario en el que se mejora la calidad o aumenta la cantidad de un bien o servicio ambiental, que en términos de utilidad se expresa de la siguiente manera (Riera y otros, 2005):

$$
U\left(p, y, r^{0}\right)=U\left(p, y-V e, r^{1}\right)
$$

Donde la utilidad (U) es función de los precios (p), del nivel de renta (y), de la cantidad o calidad del bien ambiental ( $r$ ) en su estado inicial (o) o final (1) y la variación equivalente (Ve). Ve indica la máxima cantidad de dinero que el consumidor estaría dispuesto a pagar (DAP) para que se diera una mejora en r. Por ejemplo, en 
el caso que aquí nos ocupa, la pérdida de utilidad experimentada por una persona dispuesta a pagar por la recuperación ambiental del río Segura se vería compensada por la mejora de la calidad del ecosistema.

Conocer las variables que influyen en la DAP de un individuo aporta información adicional a la toma de decisiones. Dichas variables representan las características socioeconómicas del individuo (por ejemplo, nivel de renta o estudios), pero también su grado de sensibilidad ambiental o su relación con el activo objeto de la valoración. En otras palabras: la modelización de la DAP permite conocer sobre qué variables es posible actuar para incrementar la aceptabilidad social de las actuaciones de mejora ambiental. Asimismo, permite comprobar si las respuestas de los individuos sobre su DAP se ajustan o no a la teoría económica. Por ejemplo, a mayor renta, la disposición a pagar debería ser mayor.

En este trabajo la modelización de la DAP por la recuperación ambiental del río Segura se lleva a cabo mediante dos modelos básicos. Por un lado, un modelo logit multivariante de variable dependiente binaria, que permite calcular la probabilidad de que un individuo esté o no dispuesto a pagar por la mejora propuesta (Menard, 2002); por otro lado, se ha empleado un modelo tobit censurado en cero para la modelización de la DAP, el cual tiene en cuenta que la variable DAP no presenta valores negativos. Este modelo predice la DAP máxima de un individuo en función de otras variables explicativas (Amemiya, 1984).

\section{CASO DE ESTUDIO: EL RÍO SEGURA EN SU TRAMO OJÓS-CONTRAPARADA}

El área de estudio se sitúa en uno de los tramos medios del río Segura, conocido como Ojós-Contraparada. Sus 34 km de longitud atraviesan nueve municipios, hasta su llegada al conocido como azud mayor de la Contraparada del río Segura. La figura 2 muestra la localización del tramo estudiado.

Este tramo ha sido degradado por la actividad del hombre a lo largo de décadas, como resultado de una gestión que ha ignorado la capacidad del río de proveer bienes y servicios ambientales. Así, el crecimiento demográfico, la actividad industrial, la operación de presas situadas aguas arriba y la derivación de aguas para riego han sido las principales presiones que han provocado la contaminación de las aguas del río y el deterioro de sus riberas.

A partir de los municipios de Ceutí y Lorquí este tramo presenta el mayor nivel de degradación (CHS, 2007). Por este motivo la Confederación Hidrográfica del Segura (CHS), principal gestor del río, ha diferenciado dos masas de agua distintas en la zona, como se muestra en la tabla 2. 
Figura 2. Localización del tramo de estudio

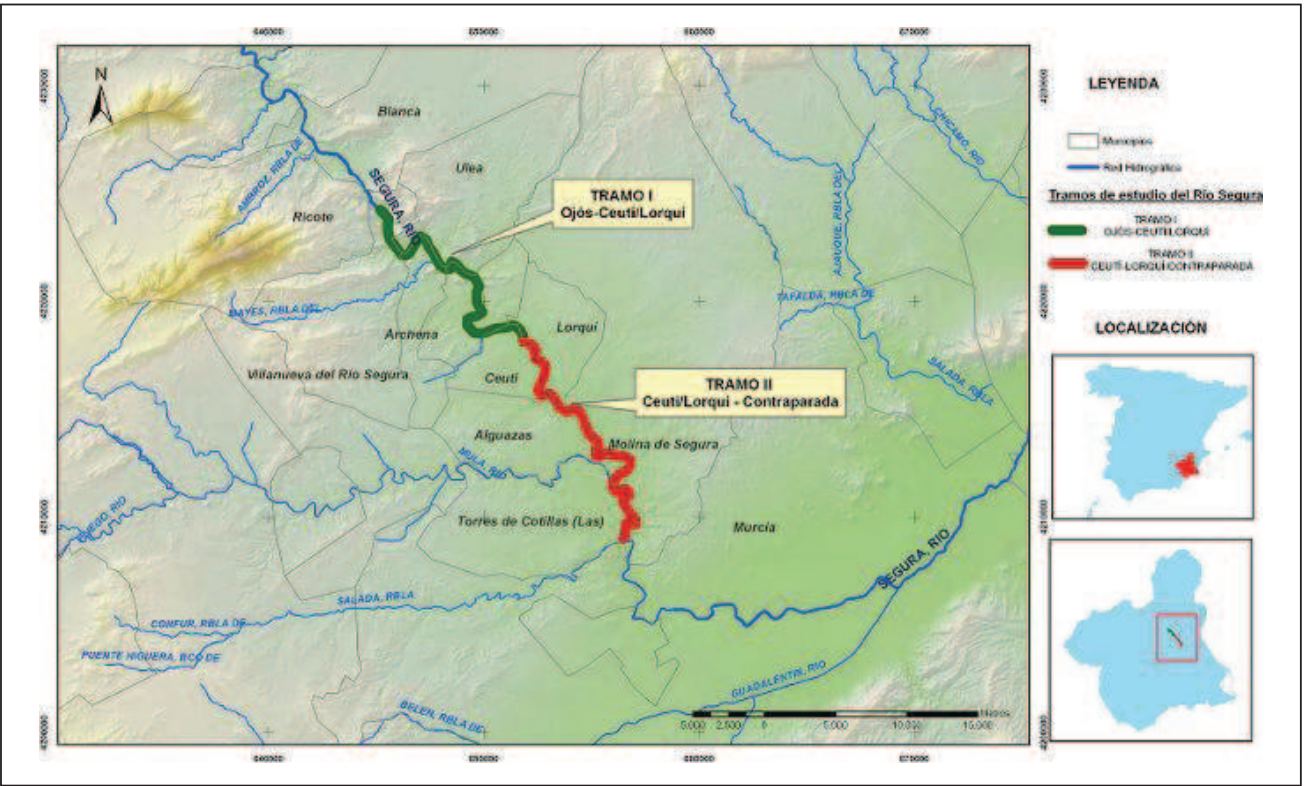

Fuente: elaboración propia.

El caudal ecológico mínimo establecido por la CHS para asegurar el mantenimiento de los ecosistemas fluviales y la dilución de vertidos es de $3 \mathrm{~m}^{3} / \mathrm{s}$ para el tramo Ojós-Contraparada. No obstante, a pesar de los avances en depuración de aguas residuales, en ocasiones tienen lugar episodios de contaminación a causa de vertidos no autorizados, que merman los ecosistemas ribereños y afectan la flora y la fauna de la zona. Además, la inexistencia de un bosque de ribera en un buen estado de conservación favorece la expansión de especies invasoras que compiten con las autóctonas y que modifican el medio fluvial (figura 3), como es el caso del Arundo donax (CHS, 2008).

Tabla 2. Estado ecológico del tramo Ojós-Contraparada

\begin{tabular}{|l|c|c|c|c|}
\hline \multicolumn{1}{|c|}{ Nombre masa } & $\begin{array}{c}\text { Estado } \\
\text { biológico }\end{array}$ & $\begin{array}{c}\text { Estado } \\
\text { hidromorfológico }\end{array}$ & $\begin{array}{c}\text { Estado } \\
\text { físico-químico }\end{array}$ & $\begin{array}{c}\text { Estado } \\
\text { ecológico }\end{array}$ \\
\hline $\begin{array}{l}\text { Río Segura desde Ojós a Ceu- } \\
\text { tí-Lorquí (10669) }\end{array}$ & Bueno & Bueno & Bueno o sup. & Bueno \\
\hline $\begin{array}{l}\text { Río Segura desde Ceutí-Lorquí } \\
\text { hasta Contraparada (10668) }\end{array}$ & Malo & Malo & $\begin{array}{c}\text { Moderado o } \\
\text { inferior }\end{array}$ & Malo \\
\hline
\end{tabular}

Fuente: elaboración propia con información de CHS (2008, pp. 73-74). 
Figura 3. Río Segura en su tramo Ojós-Contraparada

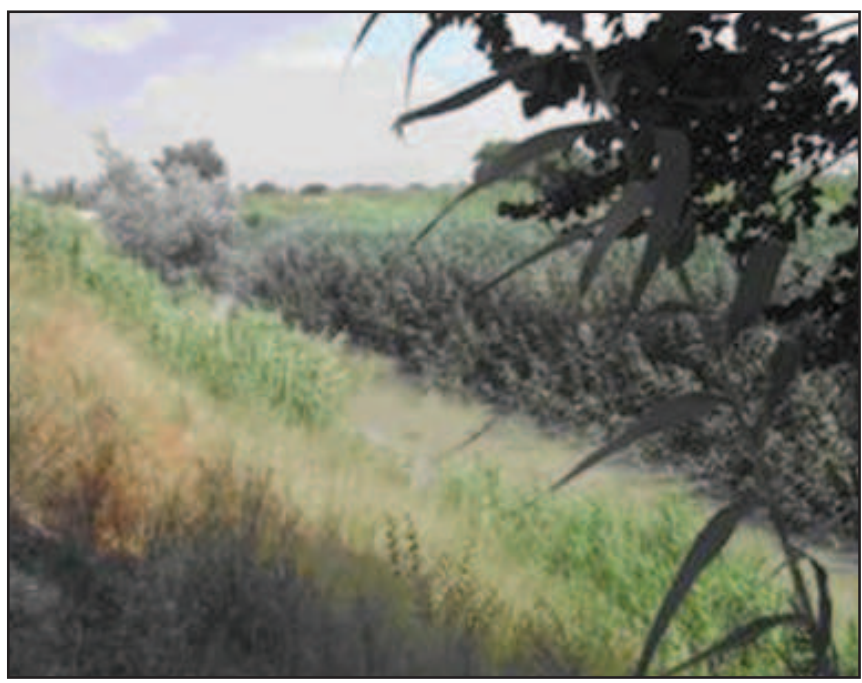

Fuente: elaboración propia.

Esta realidad ha propiciado que este tramo del río Segura sea objeto de distintos planes de gestión de recursos hídricos, con el fin de recuperar sus elementos naturales y culturales. En este sentido, en el Anexo II del Plan Hidrológico Nacional se establece un listado de actuaciones para el período 2000-2008, que en el caso de la Confederación Hidrográfica del Segura se contempla la Recuperación ambiental río Segura entre Ojós y Contraparada (BOE, 2001). Por su parte el programa AGUA también considera una serie de medidas de mejora de la calidad del agua, prevención de inundaciones y restauración ambiental, en la que se recoge la terminación de la recuperación ambiental del río Segura entre Ojós y Contraparada (BOE, 2004), ejemplos de las cuales pueden apreciarse en la figura 4. Las actuaciones se resumen en las siguientes (ACUAMED, 2006):

- Generación y señalización de rutas y caminos para potenciar y dar a conocer a la población el conjunto de elementos de interés.

- Comunicación de las distintas áreas mediante una serie de rutas que se seña lizarán aprovechando para ello los caminos y sendas existentes.

- Revegetación del soto con especies arbóreas y arbustivas características de ribera, llevada a cabo de una forma natural.

- Actuación sobre sotos existentes para favorecer la creación de manchas de vegetación riparia. 
- Restauración de elementos hidráulicos tradicionales, como norias, con el objeto de favorecer el conocimiento de este tipo de infraestructuras.

Figura 4. Actuaciones de recuperación ambiental (revegetación de sotos)

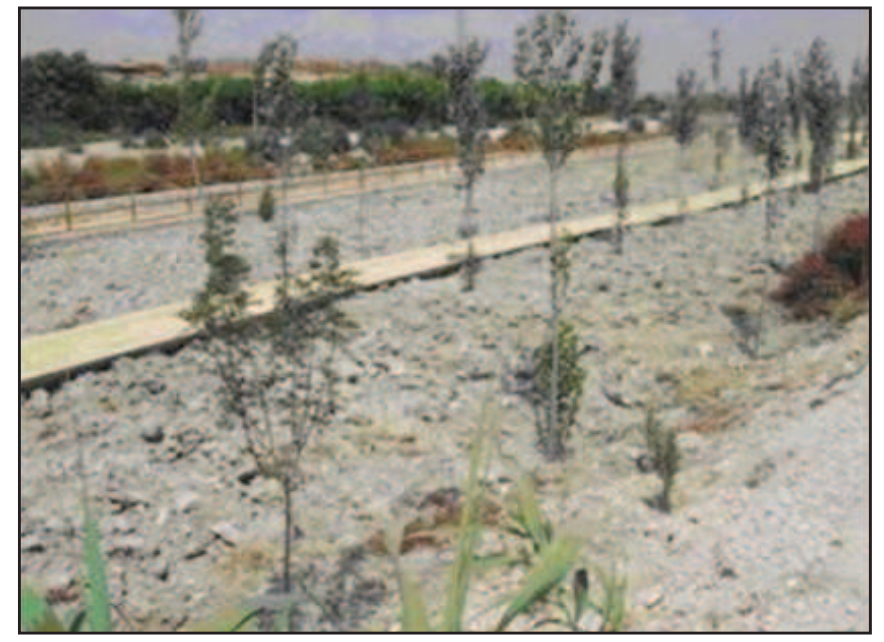

Fuente: elaboración propia.

\section{DISEÑO DE LA VALORACIÓN CONTINGENTE}

La aplicación del método de la VC se basa en la realización de encuestas a los beneficiarios potenciales del activo ambiental que se pretende valorar. Para conocer más sobre el diseño de un trabajo de VC se recomienda la consulta de Riera $y$ otros (2005). A continuación se presenta las características propias del ejercicio VC diseñado para este trabajo.

El cuestionario utilizado cuenta con un total de 27 preguntas repartidas en 5 bloques y fue redactado a partir de los resultados de un cuestionario piloto realizado a 20 individuos en abril de 2009. Algunas de las preguntas estaban dirigidas a determinar el grado de concienciación ambiental de cada individuo y su vínculo con el río Segura y, además, a recopilar aquella información socioeconómica relevante para la modelización de la disposición a pagar. Las preguntas relativas a la valoración económica del tramo Ojós-Contraparada se realizaron mediante un formato mixto (Pearce y Özdemiroglu, 2002), en el que el individuo debía contestar si estaría dispuesto o no a pagar (formato dicotómico o binario) y cuánto estaría dispuesto a pagar como máximo (formato abierto) por la recuperación ambiental del tramo Ojós-Contraparada. Dicho pago se articuló mediante un incremento en el recibo del agua repartido a lo largo del año. 
Durante las entrevistas los individuos eran informados sobre el escenario de la valoración mediante un folleto informativo compuesto de imágenes y texto. En él se describía el estado actual del río, las actuaciones previstas y el resultado de las mismas, como se describió en la sección anterior. De esta manera los individuos valoraron el cambio de la calidad ambiental en términos de uso (recreo) y no uso (mejora de la biodiversidad y del patrimonio cultural).

El proceso de encuesta tuvo lugar a través de entrevistas personales realizadas a los habitantes mayores de 18 años de las comarcas ribereñas del río Segura. Así, la población objetivo quedó constituida por un total de 901.828 habitantes (INE, 2009). La muestra se obtuvo a partir de un muestreo aleatorio simple, asegurando la representatividad de las variables socioeconómicas tales como edad, sexo y nivel de estudios. Finalmente, se realizaron 400 encuestas durante los meses de mayo y junio de 2009. Dada la naturaleza dicotómica de la variable disposición a pagar (sí/no) y que la población objetivo tiende a infinito, el error de muestreo se sitúa entre el 3\% ( $\mathrm{p}=\mathrm{q}=0.5)$ y el $5 \%(\mathrm{p}=0.1 ; \mathrm{q}=0.9)$ para un nivel de confianza del 95\% (Weisberg, 2005).

\section{RESULTADOS}

El análisis descriptivo de las respuestas sobre la aceptación del pago permite construir y explicar el mercado hipotético planteado durante el proceso de encuesta. En primer lugar, el número de individuos que declararon estar dispuestos a pagar por la mejora prevista para el tramo Ojós-Contraparada fue de 217, un 54.3\% de la muestra. El 45.7\% restante se puede dividir en dos grupos de negación (Riera y otros, 2005): por un lado están aquellas respuestas consideradas como ceros reales, dadas por individuos que no están dispuestos a pagar porque no consideran necesarias las actuaciones propuestas, o bien, porque no disponen de renta suficiente para sufragarlas; por otro lado, están aquellos individuos que arguyen que los esfuerzos económicos para la mejora del río Segura deben correr a cargo de la Administración Pública o mediante una vía distinta al incremento en la tarifa del agua. Este último grupo constituye el grupo de los ceros protesta, es decir, individuos que no están de acuerdo con el ejercicio planteado y cuya respuesta podría ser positiva si la valoración se planteara con un enfoque diferente. Sea como fuere, este grupo no participa en el mercado y, por tanto, este quedaría constituido por 288 individuos, como se muestra en la figura 5. La DAP media en el mercado hipotético es de 25.03 €/año1. Por otra parte, el valor de la DAP mediana es $20 € /$ año, cercano al valor medio, lo que pone de manifiesto que no se presentaron valores atípicos en los resultados de la encuesta (Sepúlveda-Vargas, 2008).

$1 €=1.3 \$$ USA (Fuente: Banco Central Europeo, 2012) 
Figura 5. Composición final del mercado hipotético

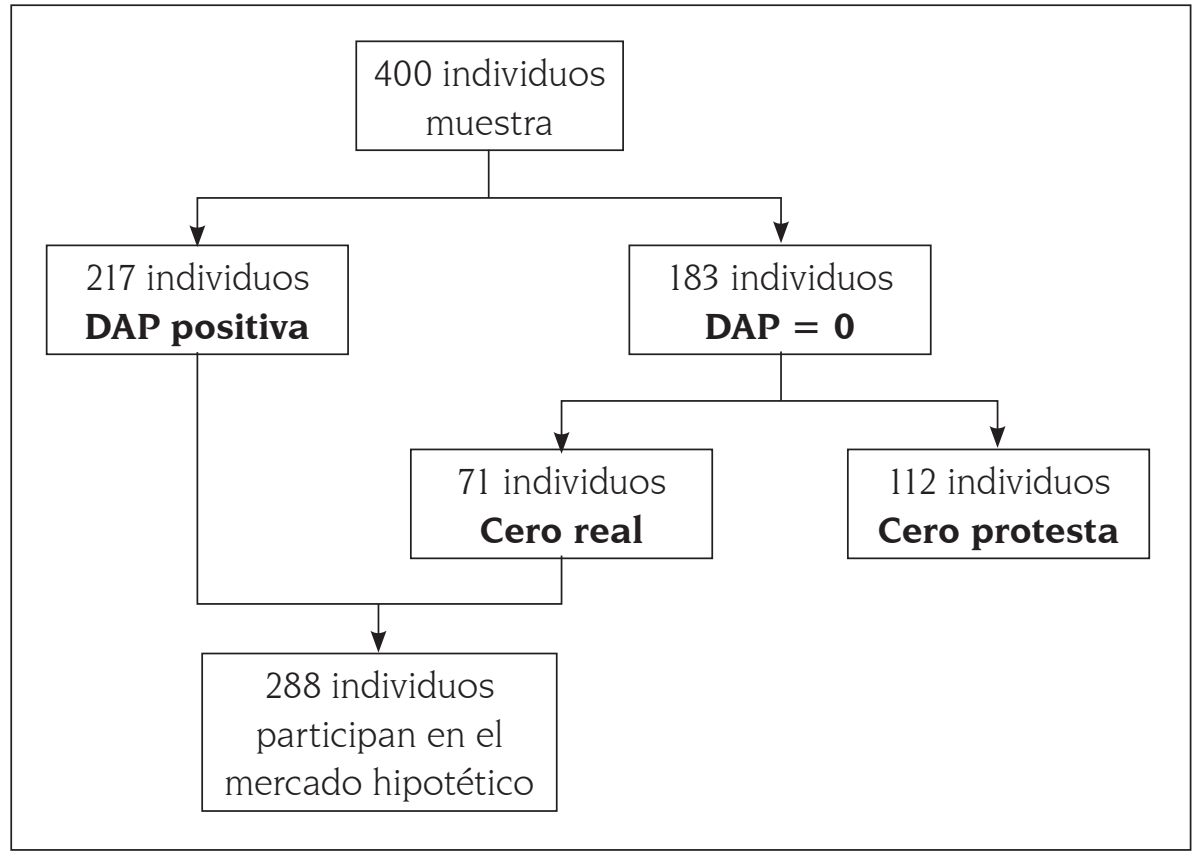

Fuente: elaboración propia.

La modelización de la respuesta a la pregunta dicotómica (sí/no) sobre la DAP se ha realizado mediante un modelo logit multivariante de variable binaria (DAPB), a partir de los 288 individuos que forman parte del mercado hipotético ${ }^{2}$ (tabla 3).

El modelo estima la probabilidad de una respuesta positiva a la pregunta dicotómica sobre la DAPB en función de variables socioeconómicas como renta, estudios y si es usuario del tramo, es decir, si ha visitado el tramo al menos una vez en los dos últimos años. Se han incluido también variables psicográficas que hacen referencia al nivel de compromiso ecológico afectivo (ICA) y verbal (ICV) de cada encuestado (Maloney, Ward y Braucht, 1975; Castro, 2000). Así, un usuario con altos niveles de compromiso ecológico y estudios universitarios tendría una alta intención de pago. Además, la probabilidad de una disposición al pago positiva se incrementa a medida que aumenta la renta familiar.

2 El lector interesado en solicitar el acceso a los datos utilizados en la modelización puede ponerse en contacto con angel.perni@um.es 
A. Perni • J. M. Martínez-Paz

Tabla 3. Modelo de la intención de pago (DAPB)

\begin{tabular}{|l|c|c|c|}
\hline \multicolumn{1}{|c|}{ Variable } & Parámetro $\beta$ & Test de Wald & Significación \\
\hline ICA (1-5) & 0.534 & 5.879 & 0.015 \\
\hline ICV (1-5) & 0.360 & 3.008 & 0.083 \\
\hline Renta Familiar (103 €/mes) & 0.293 & 5.301 & 0.021 \\
\hline Universitario (Sí = 1; No =0) & 1.330 & 13.424 & 0.000 \\
\hline Usuario (Sí = 1; No =0) & 1.314 & 12.822 & 0.000 \\
\hline Constante & -4.197 & 18.306 & 0.000 \\
\hline $\begin{array}{l}\text { Porcentaje de clasificación correcta (PCC) }=82.6 \% \\
\text { Contraste de razón de verosimilitudes: Chi-cuadrado(5) }=138.5 \text { [0.0000] }\end{array}$ \\
\hline
\end{tabular}

Fuente: elaboración propia

Para conocer qué variables determinan la cuantía máxima que un individuo estaría dispuesto a pagar (DAPT) se ha estimado un modelo de regresión con es pecificación tobit censurado en cero (tabla 4).

Tabla 4. Modelo de la disposición máxima a pagar (DAPT)

\begin{tabular}{|l|c|c|c|}
\hline \multicolumn{1}{|c|}{ Variable } & Parámetro $\beta$ & Z & Significación \\
\hline ICV (1-5) & 7.502 & 3.565 & 0.000 \\
\hline Universitario (Sí = 1; No = 0) & 16.196 & 4.153 & 0.000 \\
\hline Renta Familiar (€/mes) & 0.003 & 3.024 & 0.003 \\
\hline Usuario (Sí = 1; No = 0) & 6.134 & 1.747 & 0.081 \\
\hline Localidad en el tramo (Sí = 1; No =0) & 8.700 & 1.833 & 0.067 \\
\hline Constante & -44.687 & -3.702 & 0.000 \\
\hline $\begin{array}{l}\text { Pseudo } \mathrm{R}^{2}=0,158 \\
\text { Contraste de razón de verosimilitudes: Chi-cuadrado(5) }=108.3[0.0000]\end{array}$ \\
\hline
\end{tabular}

Fuente: elaboración propia.

Dado el carácter lineal de esta función de demanda, se puede concluir que un punto adicional de ICV resulta en un incremento de $7.5 €$, mientras que un universitario pagaría $16 €$ más que un no universitario. La condición de usuario del río o 
Valoración económica de los beneficios ambientales de la recuperación del río Segura (España)

residente en algunas de las localidades por las que discurre el tramo Ojós-Contraparada es también motivo de disposiciones al pago mayores. En cuanto a la renta familiar, por cada $1,000 € /$ mes se incrementaría la DAPT en $3 €$.

De la interpretación de ambos modelos econométricos se deduce que el desarrollo de programas de educación ambiental en el río Segura se traduciría en un aumento de la probabilidad y del monto de la disposición a pagar de los ciudadanos, es decir, de la aceptabilidad de este tipo de actuaciones, debido a su efecto sobre las variables ICA e ICV.

Una vez caracterizada la demanda de la recuperación ambiental del río Segura, se pasa a estimar en términos monetarios los beneficios ambientales asociados al cambio de estado. Dicha estimación se lleva a cabo agregando la DAP media por el total de hogares del área de estudio, dado que el vehículo de pago fue el recibo del agua (Ramajo-Hernández y Saz-Salazar, 2012). A partir de la DAP media obtenida de 25.03 €/año, la población objetivo de 901,828 individuos y el número medio de habitantes por hogar de 3.15 (INE, 2007), se obtiene que la renta ambiental generada por la mejora propuesta en las actuaciones proyectadas en la zona alcanzaría los $7,165,954$ €/año.

Tabla 5. Disposición a pagar de usuarios y no usuarios (€/año)

\begin{tabular}{|l|l|c|c|c|}
\hline \multicolumn{2}{|c|}{} & N & Media & Desv. típ. \\
\hline \multirow{4}{*}{ DAPT } & DAP Usuarios & 125 & 26.29 & 21.41 \\
\cline { 2 - 5 } & DAP No Usuarios & 163 & 24.07 & 24.60 \\
\cline { 2 - 5 } & Total & 288 & 25.03 & 23.26 \\
\hline
\end{tabular}

Fuente: elaboración propia.

Este valor de renta ambiental se puede dividir en sus fracciones de uso y no uso. Para ello, se calcula la DAP media de usuarios (DAPU) y no usuarios (DAPNU), mostradas en la tabla 5. El valor de no uso viene dado por el valor de la DAPNU multiplicada por el número de hogares, lo que arroja una renta ambiental de no uso de 6,891,111 €/año. Finalmente, la renta ambiental de uso viene dada por el incremento de la DAPU respecto a la DAPNU (2.22 €/año) extrapolado a la población de usuarios (43\% según datos de la encuesta), lo que resulta en un valor de 273,297 €/año.

\section{CONCLUSIONES}

La gestión integrada de las masas de agua propuesta en la DMA se ha traducido en el establecimiento de un enfoque multidisciplinar para la consecución del objetivo del 
buen estado. Así, el papel otorgado por esta Directiva al Análisis Económico necesita todavía de un mayor desarrollo. En este sentido, los proyectos realizados en aras de la recuperación de las masas de agua deben evaluarse desde una perspectiva económica. Por tanto, es imprescindible la valoración de los beneficios ambientales derivados de dichas actuaciones.

Este trabajo ha estimado en términos monetarios los beneficios ambientales obtenidos de la recuperación ambiental del río Segura (España). Se ha aplicado el método de la VC para estimar los beneficios ambientales de la recuperación del río Segura en su tramo Ojós-Contraparada. Los resultados muestran que una alta proporción de ciudadanos, el 54.3\%, estarían dispuestos a pagar por este tipo de proyectos, con una DAP promedio de $25 €$ /año. A partir de este resultado se ha estimado una renta ambiental del orden de los siete millones de euros anuales. Asimismo, se ha identificado que variables como el grado de concienciación ambiental y el nivel de estudios influyen de manera positiva en la disposición al pago. De esta manera, programas de educación y voluntariado ambiental en el río Segura se traducirían en un aumento del monto de la disposición a pagar de los ciudadanos, es decir, un aumento de la aceptabilidad social de las actuaciones.

Finalmente, el valor de renta ambiental presentado debe ser considerado en los análisis costo-beneficio de las actuaciones emprendidas en el río Segura como beneficio ambiental de la recuperación de sus bienes y servicios, con el fin de justificar desde una óptica económica la rentabilidad social de este tipo de proyectos.

\section{BIBLIOGRAFÍA}

ACUAMED (2006). Informe de viabilidad de la actuación 2.3.b terminación de la recuperación ambiental del Segura entre Ojós y Contraparada (Murcia). Aguas de las Cuencas Mediterráneas S. A.

Amemiya, T. (1984). Tobit models - A survey. En: Journal of Econometrics, Vol. 24, N. 1-2, pp. 3-61.

Azqueta, D. (1997). Valoración económica de la calidad ambiental. Madrid, McGraw-Hill, 299p.

Azqueta, D. (2002). Introducción a la Economía Ambiental. Madrid, McGraw-Hill, 456p.

Barbier, E. B.; Acreman, M. y Knowler, D. (1997). Valoración económica de los humedales. Guía para decisores y planificadores. Gland (Suiza), Oficina de la Convención de Ramsar, 155p.

Bateman, I. J.; Brouwer, R.; Davies, H.; Day, B.H.; Deflandre, A.; Falco, S.D.; Georgiou, S.; Hadley, D.; Hutchins, M.; Jones, A.P.; Kay, D.; Leeks, G.; Lewis, M.; Lovett, A.A.; Neal, C.; Posen, P.; Rigby, D. y Turner, R.K. (2006). Analysing the Agricultural Costs and 
Valoración económica de los beneficios ambientales de la recuperación del río Segura (España)

Non-market Benefits of Implementing the Water Framework Directive. En: Journal of Agricultural Economics, Vol. 57, N. ${ }^{\circ}$ 2, pp. 221-237.

Birol, E.; Karousakis, K. y Koundouri, P. (2006). Using economics methods to inform water resource management policies: a survey and critical appraisal of available methods and an application. En: Science of the Total Environment, Vol. 365, N.․ 1-3, pp. 105-122.

BOE (2001). Ley 10/2001, de 5 de julio, del Plan Hidrológico Nacional. Madrid, Boletín Oficial del Estado, 23p.

BOE (2004). Real Decreto Ley 2/2004, de 18 de junio, por el que se modifica la Ley 10/2001, de 5 de julio, del Plan Hidrológico Nacional. Madrid, Boletín Oficial del Estado, 5p.

Bouleau, G. (2008). The WFD dreams: between ecology and economics. En: Water and Environment Journal, Vol. 22, N. ${ }^{\circ}$, pp. 235-240.

Brauman, K.A., Daily, G.C., Duarte, T.K. and Mooney, H.A. (2007). The Nature and Value of Ecosystem Services: an Overview Highlighting Hydrologic Services. En: The Annual Review of Environment and Resources, Vol. 32, pp. 67-98.

Brouwer, R. (2004). The value of Clean Water. Perception and Valuation of the Benefits Asociated with the Implementation of the Water Framework Directive in the Netherlands. RIZA rapport 2004-013. RIZA, Lelystad, The Netherlands.

Brox, J.A., Kumar, R., y Stoltery, K. (2003). Estimating Willingness to Pay for Improved Water quality in the Presence of Item non-response Bias. En: American Journal of Agricultural Economics, Vol. 85, N. ${ }^{\circ}$ 2, pp. 415-429.

Castro, R. (2000). Naturaleza y funciones de las actitudes ambientales. En: Estudios de Psicología, Vol. 22, N.․ 1, pp. 11-22.

Cardoso, A. y Benhin, J. (2011). Assessing the viability of protecting Colombian Caribbean coast from sea level rise: an economic valuation approach. Semestre Económico, Vol. 29, N. 14 , pp. 13-30.

CHS (2007). Estudio general de la Demarcación Hidrográfica del Segura. Confederación Hidrográfica del Segura. Madrid, Ministerio de Medio Ambiente, Rural y Marino, 359p.

CHS (2008). Esquema provisional de temas importantes. Confederación Hidrográfica del Segura. Madrid, Ministerio de Medio Ambiente, Rural y Marino, 188p.

Ciriacy-Wantrup, S. (1952). Resource conservation; economics and policies. Berkeley, University of California Press, 395p.

Comisión Europea (2000). Directive 2000/EC of the European Parliament and of the Council of 23 October 2000 Establishing a Framework for Community Action in the Field of Water Policy Official Journal 22 December 2000 L 327/1, Brussels, European Commission, 72p. 
Comisión Europea (2003). Economics and the Environment: The implementation Challenge of the Water Framework Directive. A Guidance Document. Brussels, European Commission, 274p.

Cooper, P., Poe, G.L y Bateman I.J. (2004). The structure of motivation for contingent values: A case of study of lake water quality improvement. En: Ecological Economics, Vol. 50, N. ${ }^{\circ} 1-2$, pp. $69-82$.

Del Saz-Salazar, S., Hernández Sancho F. y Sala Garrido, R. (2008). Estimación del valor económico de la calidad del agua de un río mediante una doble aproximación: una aplicación de los principios económicos de la Directiva Marco del Agua. En: Economía Agraria y Recursos Naturales, Vol. 9, N. ${ }^{\circ}$ 1, pp. 37-63.

EEA (2009). Water resources across Europe - confronting water scarcity and drought. Report No 2/2009. Denmark, European Environment Agency, 60p.

EEA (2010). Scaling up ecosystem benefits. A contribution to The Economics of Ecosystems and Biodiversity (TEEB) study. Report. N. ${ }^{\circ}$ 4/2010. Denmark, European Environment Agency, 44p.

England, J.; Skinner, K.S y Carter, M.G. (2008). Monitoring, river restoration and the Water Framework Directive. En: Water and Environment Journal, Vol. 22, N. ${ }^{\circ}$ 4, pp. 227-234.

Fianko, J.R.; Osae, S. y Achel, D. (2008). Impact of anthropogenic activities on the Densu River in Ghana. En: Water and Environmental Journal, Vol. 23, N. ${ }^{\circ}$ 3, pp. 229-234.

Freeman, A. M. (2003). The Measurement of Environmental and Resource Values: Theory and Methods. Washington DC, Resources for the Future, 496p.

Hein, L.; Van Koppen, K.; de Groot, R.S. y Van Lerland, E.C. (2006). Spatial scales, stakeholders and the valuation of ecosystem services. En: Ecological Economics, Vol. 57, N. ${ }^{\circ}$ 2, pp. 209-228.

INE (2007). Censo de Población y Viviendas 2001. Resultados definitivos. [En línea]. España, Instituto Nacional de Estadística. Disponible en: $\square$ http://www.ine.es/censo accesible/ es/consulta.jsp] [26-09-2012]

INE (2009). Revisión del padrón municipal de 2008. [En línea]. España, Instituto Nacional de Estadística. Disponible en: Dhttp://www.ine.es/inebmenu/mnu cifraspob.htm]. [12-03-2010]

Ivesa, L.; Lyons, D.M. y Devescovi, M. (2009). Assessment to the ecological status of north-eastern Adriatic coastal Waters (Istria, Croatia) using macroalgal assemblages for the European Union Water Framework Directive. En: Aquatic Conservation: Marine and Freshwater Ecosystems, Vol. 19, N. ${ }^{\circ}$ 1, pp. 14-23.

Klessig, L. (2001). Lakes and society: The contribution of lakes to sustainable societies. En: Lakes \& Reservoirs: Research and Management, Vol. 6, N. ${ }^{\circ}$ 2, pp. 95-101. 
Valoración económica de los beneficios ambientales de la recuperación del río Segura (España)

Lant C. y Mullens B. (1992). Lake and river quality for recreation management and contingent valuation. En: Journal of the American Water Resources Association, Vol. 27, N. ${ }^{\circ} 3$, pp. 453-460.

Maloney, M.; Ward, M. y Braucht, G. (1975). Psychology in action: A Revised Scale for the Measurement of Ecological Attitudes and Knowledge. En: American Psychologist. Vol. 30, N. ${ }^{\circ}$, pp. $787-790$.

Martín-Ortega, J. (2009). Los beneficios ambientales de las aguas del Guadalquivir: un análisis económico. Fundación Pública Andaluza, Centro de Estudios Andaluces, Consejería de la Presidencia, Junta de Andalucía, 226p.

Martín-Ortega, J. (2013). Economic prescriptions and policy applications in the implementation of the European Water Framework Directive. Environmental Sciences \& Policy. En prensa: 〈http://dx.doi.org/10.1016/j.envsci.2012.06.002〉

Menard, S. (2002). Applied logistic regression analysis. Series: Quatitative Applications in the Social Sciences 106. Londres, Sage Publications, 128p.

Mitchell, R. y Carson, R. (1989). Using Surveys to Value Public Goods: the Contingent Valuation Method. Washington DC, Resources for the Future, 488p.

Moreno-Sanchez, R.; Maldonado, J.H.; Wunder, S. y Borda-Almanza, C. (2012). Heterogeneous users and willingness to pay in an ongoing payment for watershed protection initiative in the Colombian Andes. En: Ecological Economics, Vol. 75, pp. 126-134.

Mouton, A.M.; Most, H. van der; Jeuken, A.; Goethals, P.L.M. y Pauw, N. de. (2009). Evaluation of river basin restoration options by the application of the Water Framework Directive Explorer in the Zwalm River Basin (Flanders, Belgium). En: River Resesarch and Applications, Vol. 25, N. ${ }^{\circ}$ 1, pp. 82-97.

Newson, M.D. y Large, A.R.G. (2006). 'Natural' rivers, 'hydromorphological qualiqty' and river restoration: a challenging new agenda for applied fluvial geomorphology. En: Earth Surface Processes and Landforms, Vol. 31, N.․ 13, pp. 1606-1624.

Ortiz, J.L. (2002). La directiva marco del agua (2000/60/CE): aspectos relevantes para el proyecto GUADALMED. En: Limnética, Vol. 21, N. 3-4, pp. 5-12.

Pearce, D. y Özdemiroglu, E. (2002). Economic valuation and stated preference techniques. London, Local Transport Department, 87p.

Pearce, D. y Turner, W. (1995). Economía de los recursos naturales y del medio ambiente. Madrid, Celeste Ediciones-Colegio de Economistas de Madrid, 448p.

Perni, A.; Martínez-Carrasco, F. y Martínez-Paz, J.M. (2011). Economic valuation of coastal lagoon environmental restoration: Mar Menor (SE Spain). En: Ciencias Marinas, Vol. 37, N. ${ }^{\circ}$, pp. $175-190$.

Semestre Económico, volumen 15, №. 32, pp. 15-40 • ISSN 0120-6346, julio-diciembre de 2012, Medellín, Colombia 
A. Perni • J. M. Martínez-Paz

Ramajo-Hernandez, J. y del Saz-Salazar, S. (2012). Estimating the non-market benefits of water quality improvement for a case study in Spain: A contingent valuation approach. En: Environmental Science E Policy, Vol. 22, pp. 47-59.

Riera, P.; Garcia, D.; Kristron, B. y Brannlund, R. (2005). Manual de economía ambiental y de los recursos naturales. Madrid, Editorial Thomson, 355p.

Sari, M.; Kadioglu, M.; Arabaci, M. y Ertan, A. (2003). Ecological sharing of water for healthy management of fisheries and irrigation under drought conditions in Bend-I Mahi River, Van, Turkey. En: Journal of Environmental Protection and Ecology, Vol. 4, N. ${ }^{\circ}$ 1, pp. 166- 178.

Schaafsma, M. y Brouwer, R. (2006). Overview of Existing Guidelines and Manuals for the Economic Valuation of Environmental Costs and Benefits. AquaMoney Project Working Document.

Sepúlveda-Vargas, (2008). Valoración económica del uso recreativo del Parque Ronda del Sinú, en Monteria, Colombia. Semestre Económico, Vol. 11, N.ํ22, pp. 67-90.

Skinner, K.S. y Bruce-Burgess, L. (2007). Strategic and Project level river restoration protocols - Key components for meeting de requirements of the Water Framework directive (WFD). En: Water and Environment Journal, Vol. 19, N. ${ }^{\circ}$ 2, pp. 135-142.

Strosser, P. (2006). Assessing Environmental Costs in the Context of the Implementation of the European Union Framework Directive. Hydronomic Convention. Barcelona, 13-14 Noviembre.

Weisberg, H. (2005). The Total Survey Error Approach. Chicago, University of Chicago Press, 400p. 


\section{ANEXO FORMATO DE ENCUESTA CUESTIONARIO DE LA VALORACIÓN ECONÓMICA DE LA RECUPERACIÓN AMBIENTAL DEL RÍO SEGURA}

CUESTIONARIO n ${ }^{\circ}$

FECHA _ / _ I

/ LUGAR

"Buenos días (tardes o noches), si es tan amable, me gustaría que me respondiese a una serie de preguntas relacionadas con el río Segura en su tramo Ojós-Contraparada. Estoy realizando un trabajo para la Universidad de Murcia, que trata sobre la valoración económica de la recuperación de la calidad ambiental de este tramo del río. Necesito conocer su opinión al respecto.

El cuestionario es anónimo, y con el fin de que los datos sean reales y verídicos, necesito que responda con total sinceridad".

P.1) De los siguientes problemas que atañen a la sociedad en general, ¿podría indicarme, en una escala de 1 a 5, qué importancia tiene para usted cada uno de ellos?

\begin{tabular}{|l|c|c|c|c|c|}
\hline \multirow{2}{*}{} & \multicolumn{2}{|c|}{ Poco importante } & \multicolumn{2}{c|}{$\begin{array}{c}\text { Muy } \\
\text { importante }\end{array}$} \\
\cline { 2 - 6 } & 1 & 2 & 3 & 4 & 5 \\
\hline $\begin{array}{l}\text { Problemas sociales (terrorismo, inseguridad } \\
\text { ciudadana, inmigración ilegal, drogas...) }\end{array}$ & & & & & \\
\hline $\begin{array}{l}\text { Deterioro del medioambiente (cambio climático, } \\
\text { contaminación, pérdida de biodiversidad...) }\end{array}$ & & & & & \\
\hline $\begin{array}{l}\text { Situación económica actual (carestía de la vida, } \\
\text { desempleo, vivienda...) }\end{array}$ & & & & & \\
\hline Otros: & & & & & \\
\hline
\end{tabular}

P.2) De los siguientes problemas ambientales, ¿podría indicarme qué importancia tiene para usted cada uno de ellos?

\begin{tabular}{|l|c|c|c|c|c|}
\hline \multirow{2}{*}{} & \multicolumn{2}{|c|}{$\begin{array}{c}\text { Poco } \\
\text { importante }\end{array}$} & & \multicolumn{3}{c|}{$\begin{array}{c}\text { Muy } \\
\text { importante }\end{array}$} \\
\cline { 2 - 6 } & 1 & 2 & 3 & 4 & 5 \\
\hline Contaminación atmosférica y acústica & & & & & \\
\hline Cambio climático & & & & & \\
\hline
\end{tabular}


A. Perni • J. M. Martínez-Paz

\begin{tabular}{|l|c|c|c|c|c|}
\hline & \multicolumn{2}{c|}{$\begin{array}{c}\text { Poco } \\
\text { importante }\end{array}$} & & \multicolumn{2}{c|}{$\begin{array}{c}\text { Muy } \\
\text { importante }\end{array}$} \\
\cline { 2 - 7 } & 1 & 2 & 3 & 4 & 5 \\
\hline Mala gestión de lo recursos hídricos & & & & & \\
\hline pérdida de biodiversidad & & & & & \\
\hline Otros: & & & & & \\
\hline
\end{tabular}

P.3) Al igual que en las dos preguntas anteriores, de los siguientes problemas relacionados con el agua, ¿podría indicarme qué importancia tiene para usted cada uno de ellos?

\begin{tabular}{|l|c|c|c|c|c|}
\hline & \multicolumn{2}{c|}{$\begin{array}{c}\text { Poco impor- } \\
\text { tante }\end{array}$} & & \multicolumn{2}{c|}{$\begin{array}{c}\text { Muy } \\
\text { importante }\end{array}$} \\
\cline { 2 - 7 } & 1 & 2 & 3 & 4 & 5 \\
\hline Vertidos de aguas residuales sin depurar & & & & & \\
\hline Sequías y escasez de agua & & & & & \\
\hline Degradación de humedales & & & & & \\
\hline Otros: & & & & & \\
\hline
\end{tabular}

P.4) En relación a las distintas Administraciones (local, autonómica y estatal) ¿cree usted que estas se preocupan lo suficiente por la conservación y protección de los ríos?

\begin{tabular}{|l|l|l|l|l|l|}
\hline & \multicolumn{3}{|c|}{ Nada } & & \multicolumn{3}{|c|}{ Mucho } \\
\hline Puntuación & 1 & 2 & 3 & 4 & 5 \\
\hline
\end{tabular}

P.5.1) ¿Cuántas veces ha visitado usted el río Segura, en su tramo Ojós-Contraparada, en el último año?

1. 2 a $5 . \quad$ Más de 5 .

Ninguna ¿Cuántos años hace que lo visitó por última vez?

Usuario. $\quad$ No usuario (〉2años) (pasar a la P.7)

P.5.2) (Solo para USUARIOS) ¿Cómo valora usted la calidad del agua de este tramo del río Segura en la actualidad?

\begin{tabular}{|l|l|l|l|l|l|}
\hline & \multicolumn{3}{|c|}{ Muy baja } & & \multicolumn{3}{|c|}{ Muy alta } \\
\hline Puntuación & 1 & 2 & 3 & 4 & 5 \\
\hline
\end{tabular}


Valoración económica de los beneficios ambientales de la recuperación del río Segura (España)

P.6) ¿Por qué motivo/s visitó usted el río?

__ Para relajarme y disfrutar del paisaje.

Para pasear y/o montar en bicicleta.

Para practicar piragüismo.

- Trabajo.

Otros motivos:

"El Río Segura a lo largo de su recorrido por la Región de Murcia tiene distintos niveles de calidad de agua. En la Vega Alta la calidad del río es buena, mientras que en la Vega Media, en la que se sitúa nuestra zona de estudio, los niveles de contaminación son superiores, por lo que su calidad se ve gravemente afectada".

P.7) ¿Conocía usted con anterioridad esta información sobre la calidad del agua del río Segura?

Sí (pasar a P.8) $\quad$ No (pasar a P. 9)

P.8) ¿Cómo se enteró?

_ A través de la prensa

_ A través de la TV/Radio

_ Motivos profesionales
_ _ A través de Internet

A través de la observación directa del río

Otra respuesta:

“Para el cumplimiento de la normativa de la Unión Europea en materia de medioambiente, la Administración está llevando a cabo proyectos de restauración de ríos, con el fin de mejorar la calidad de las aguas."

P.9) ¿Conoce los proyectos de recuperación ambiental desarrollados o que se vayan a desarrollar en el tramo Ojós-Contraparada del río Segura?

_ No, nada _ Sí, algo sé _ Sí, bastante

“Algunas de las actuaciones que se están llevando a cabo son: trabajos para incrementar la diversidad vegetal y animal del río, mejora de la calidad paisajística, rehabilitación de norias, mejora de áreas recreativas, diseño de rutas didácticas, etc."

P.10) Valore estas actuaciones de 0 (muy negativa, estoy totalmente en contra) a 10 (muy positiva, estoy totalmente a favor)

No conozco tanto las actuaciones como para dar un valor 


\section{VALORACIÓN ECONÓMICA DE LA RECUPERACIÓN AMBIENTAL DEL TRAMO OJÓS-CONTRAPARADA}

P.11) ¿Estaría usted dispuesto/a a pagar una cantidad de dinero anual, repartido en sus recibos del agua, para la recuperación ambiental del Río Segura en la zona descrita?

_ Sí (pasar a la p. 12)

__ No (pasar a la p. 14)

P.12) De acuerdo con sus posibilidades económicas ¿ipagaría cada año $€$ ?

_ Sí. Estaría dispuesto a pagar €, pero ¿cuál sería la máxima cantidad que pagaría? $€$

_ No. No estaría dispuesto a pagar __ _ entonces ¿̇cuál sería la máxima cantidad que pagaría? _— €

P.13) ¿Podría indicarme los motivos principales por los que estaría usted dispuesto a pagar tal cantidad de dinero?

__ Mi nivel de renta me permitiría pagar esa cantidad de dinero.

__ Es suficiente con esa cantidad, si todos contribuyeran.

_ Me considero un usuario habitual de la zona.

__ Para mejorar el estado de la zona (acondicionamiento, limpieza, paneles informativos).

_ Me gusta la zona.

_ Vivo cerca de la zona.

__ Estoy a favor de la conservación y restauración de ríos.

Para conservar la nutria (u otras especies).

Otros:

P. 14) (Para los que no están dispuestos a pagar) ¿Podría indicarme por qué motivo/s no estaría dispuesto a realizar ninguna aportación?:

__ Es competencia de la Administración Pública.

__ No dispongo de renta suficiente para pagar más por el recibo del agua, aunque me importa el tema.

__ Ya dono dinero para otras iniciativas ambientales.

No considero necesario conservar y mejorar la zona.

__ No considero apropiado que el pago se realice a través del recibo del agua. 
Valoración económica de los beneficios ambientales de la recuperación del río Segura (España)

A la hora de realizar una aportación, considero otros temas más prioritarios.

Otros:

\section{ÍNDICE DE COMPROMISO ECOLÓGICO}

P. 15) ¿Hasta qué punto está de acuerdo (o no) con las afirmaciones de que (1 máximo desacuerdo, 5 máximo acuerdo?:

\begin{tabular}{|c|c|c|c|c|c|}
\hline A. Compromiso ecológico afectivo & 1 & 2 & 3 & 4 & 5 \\
\hline $\begin{array}{l}\text { - Me enfurece pensar que el Gobierno no hace } \\
\text { nada para ayudar a controlar la contaminación } \\
\text { del agua. }\end{array}$ & & & & & \\
\hline $\begin{array}{l}\text { - Llego a indignarme cuando pienso sobre el } \\
\text { daño causado a las plantas y vida animal por la } \\
\text { contaminación del agua. }\end{array}$ & & & & & \\
\hline $\begin{array}{l}\text { - Nunca me ha afectado la contaminación del } \\
\text { agua y pienso que se exagera mucho sobre el } \\
\text { tema. }\end{array}$ & & & & & \\
\hline B. Compromiso ecológico verbal & 1 & 2 & 3 & 4 & 5 \\
\hline $\begin{array}{l}\text { - Estaría dispuesto a reducir mi consumo diario } \\
\text { de agua aunque fuera un inconveniente para mí. }\end{array}$ & & & & & \\
\hline $\begin{array}{l}\text { - Estaría dispuesto a participar en los programas } \\
\text { de voluntariado ambiental para la conservación } \\
\text { del río Segura que se ofrecieran en mi municipio. }\end{array}$ & & & & & \\
\hline $\begin{array}{l}\text { - Dejaría de comprar productos de empresas que } \\
\text { contaminan las aguas continentales o marinas, } \\
\text { aunque fuese un inconveniente para mí. }\end{array}$ & & & & & \\
\hline C. Compromiso ecológico real & 1 & 2 & 3 & 4 & 5 \\
\hline $\begin{array}{l}\text { - Estoy pendiente de las propuestas medioam- } \\
\text { bientales en materia de aguas que realizará el } \\
\text { partido al que voté en las últimas elecciones } \\
\text { generales. }\end{array}$ & & & & & \\
\hline $\begin{array}{l}\text { - Utilizo detergentes, jabones y otros productos } \\
\text { biodegradables. }\end{array}$ & & & & & \\
\hline $\begin{array}{l}\text { - He colaborado en programas de voluntariado } \\
\text { ambiental en algún tramo del río Segura. }\end{array}$ & & & & & \\
\hline
\end{tabular}




\section{INFORMACIÓN SOCIOECONÓMICA}

- $\quad$ Edad _ años

- Sexo:__ Hombre ___ Mujer

- Tamaño familiar (n. ${ }^{\circ}$ miembros, incluido usted) __ personas.

- $\quad$ Nivel Estudios:

Sin estudios. ___ EGB. __ BUP/FP.___ Universitario.

- $\quad$ Renta familiar mensual:

Sin ingresos. $\quad \ldots<600 €$

$-1.200-2000 € \quad-2.000-3.000 € \quad 3.000-5000 €$

$>5000 €$

- $\quad$ Situación laboral:

Sin empleo. __ Jubilado.___ Estudiante.

__ Amo/a de casa. _ Trabaja.

- $\quad$ Localidad del domicilio habitual:

_ Casco urbano __ Urbano-periférico __ Rural.

- $\quad$ ¿Posee algún tipo de $2^{a}$ residencia?:

Rural.

Costera.

Otras:

- ¿ ¿Pertenece a algún grupo ecologista?

— No _ Sí

- ¿ ¿Posee algún vínculo con el tramo Ojós-Contraparada del río Segura?

Sí _ N No

¿Cuál?

Vivo junto al río. ___ Suelo ir por la zona.

Desarrollo alguna actividad económica en la zona

¿Cuál? 